\title{
Elemental partitioning in medium Mn steel during short-time annealing: An in-situ study using synchrotron x-rays
}

\author{
Rajib Kalsar ${ }^{\mathrm{a}}$, Soheil Sanamar ${ }^{\mathrm{b}}$, Norbert Schell ${ }^{\mathrm{c}}$, H-G. Brokmeier ${ }^{\mathrm{b}}$, R. Saha ${ }^{\mathrm{d}}$, P. Ghosh ${ }^{\mathrm{d}}$, \\ A.N. Bhagat ${ }^{\mathrm{d}}$, Satyam Suwas ${ }^{\mathrm{a}, *}$ \\ ${ }^{a}$ Department of Materials Engineering, Indian Institute of Science, Bangalore 560012, India \\ ${ }^{\mathrm{b}}$ Institute of Materials Science and Engineering, Technical University Clausthal, Germany \\ ${ }^{\mathrm{c}}$ German Engineereing Materials Science Center at DESY, Helmholtz-ZentrumGeesthacht, Hamburg, Germany \\ ${ }^{\mathrm{d}}$ Tata Steel, Research and Development Division, Jamshedpur 831005, India
}

\section{A R T I C L E I N F O}

\section{Keywords:}

Synchrotron

Medium Mn steels

Inter-critical annealing

Elemental partitioning

Microstructure

\begin{abstract}
A B S T R A C T
In this study, high energy synchrotron radiation was used to perform an in-situ diffraction experiment in medium $\mathrm{Mn}$ Fe-6Mn-0.5C-1Al alloy to study the elemental partitioning and consequent austenite phase evolution at different stages, namely, during heating, holding and cooling. It has been observed that the austenite phase fraction significantly increases on annealing at the inter-critical annealing temperature and remains stable at room temperature. The austenite phase stability at room temperature is due to the rapid partitioning of manganese (Mn) and carbon $(\mathrm{C})$ during annealing. The relative change in d-spacing $(\Delta \mathrm{d} / \mathrm{d})$ during heating-cooling confirms the partitioning of alloying elements during inter-critical annealing.
\end{abstract}

\section{Introduction}

Research interests in low to medium Mn-containing twinninginduced plasticity steels (TWIP) and transformation-induced plasticity steels (TRIP) have increased in the past few years because these materials exhibit excellent combination of strength and ductility $[1,2]$. The proportion and distribution of austenite-ferrite or austenite-martensite phases play crucial role in deciding the mechanical properties of these steels. In the past few years, various processing conditions have been adopted to get the desired properties by tuning the microstructure including annealing at the inter-critical temperature (ICT) [3-6] and treatments pertaining to reverted austenitic (RA) transformation [7-11]. Among these two treatments, ICT is the most promising method for significantly stabilizing austenite at room temperature.

The ICT process comprises of short-time annealing at the intercritical temperature during which $\mathrm{Mn}$ and $\mathrm{C}$ get significantly partitioned in different phases. The partitioned elements stabilize the austenite phase at room temperature. The amounts of $\mathrm{Mn}$ and $\mathrm{C}$ that are partitioned in the austenite phase is very important as it governs the stacking fault energy (SFE) and hence influences the associated deformation mechanisms. Although this concept has been proposed and practised in recent times, the ultimate outcome of the process is obtained through a post-mortem analysis. No reports are available that clearly reveal the quantitative estimation of the austenite phase fraction at ICT in medium Mn steels and the changes, if any, during cooling. In addition, the addition of aluminium (Al) to medium Mn multi-phase steels is known to stabilize ferrite and the austenite phase [12,13]. Suh et al. [12] examined the role of $\mathrm{Al}$ addition in microstructure and mechanical properties of a Fe-5Mn-0.12C-0.5Si steel. A very clear transition from a lath-type martensite microstructure to an elongated ferrite-type microstructure was observed on increasing $\mathrm{Al}$ addition.

The aim of the present study is to clarify the presence of the austenite phase at ICT and the rapid transformation of ferrite to austenite during annealing. Moreover, the study focuses on finding out the retained austenite phase fraction after cooling down to room temperature.

\section{Experimental methodology}

The nominal chemical composition that was chosen for the present study was a Fe-6Mn-0.5C-1Al (wt.\%) alloy. The alloy was melted several times in an arc melting furnace in argon (Ar)- atmosphere to get the best possible homogenous composition throughout the sample. The composition analysis was carried out using optical emission spectroscopy (OES) and electron probe micro-analysis (EPMA). The final composition of the as-cast specimen is listed in Table 1 . The as-cast material was hot rolled at a temperature of $650{ }^{\circ} \mathrm{C}$ to obtain a thickness reduction, from $12 \mathrm{~mm}$ to $1 \mathrm{~mm}$. The hot-rolled sample ( $1 \mathrm{~mm}$ thickness) was used for in-situ experiments. The inter-critical annealing temperature was determined

\footnotetext{
* Corresponding author.

E-mail address: satyamsuwas@iisc.ac.in (S. Suwas).
} 
(a)

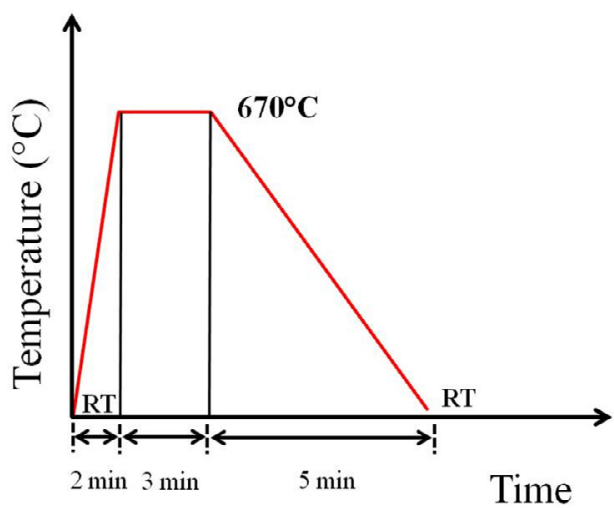

(b)

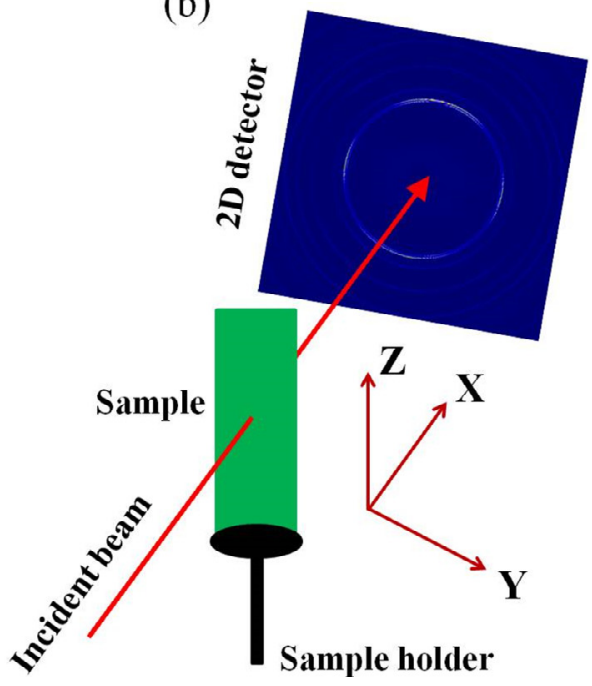

Fig. 1. (a) Heating schedule for in-situ diffraction experimental, the Y-axis indicates the heating, holding and cooling temperature, and (b) Schematic illustration shows the diffraction geometry. The flat sample was placed on a ceramic holder.
Table 1

Chemical composition (wt.\%) of the alloy used in the present investigation.

\begin{tabular}{lllll}
\hline Mn & C & $\mathrm{Al}$ & $\mathrm{Si}$ & $\mathrm{Fe}$ \\
\hline 6.5 & 0.52 & 0.94 & 0.08 & Balance \\
\hline
\end{tabular}

with the help of the Thermo-Calc software, using the TCFE7 database [14]. The ICT, that was calculated was $670{ }^{\circ} \mathrm{C}$ and corresponds to a $50 \%$ austenite phase fraction in the microstructure. The equilibrium phase fraction was calculated with respect to the composition.

The in-situ diffraction experiment was performed at the high energy beam line P07 at DESY (Hamburg, Germany). A monochromatic x-ray beam with an energy of $80 \mathrm{kV}(\lambda \sim 0.01425 \mathrm{~nm})$ and $0.5 \mathrm{~mm} \times 0.5 \mathrm{~mm}$ spot size was used for this investigation. The lattice strain and phase fractions were calculated from the diffraction patterns. A two dimensional (2D) detector was used to record the Debye-Scherrer ring patterns. The alumina $\left(\mathrm{Al}_{2} \mathrm{O}_{3}\right)$ NIST standard was used to calibrate the accurate detector distance, misalignment and wavelength. The $\theta-2 \theta$ conversion from the Debye Scherrer ring was carried using the Fit2D software [15]. Heating was done using an induction copper coil. The sample (with dimensions $15 \mathrm{~mm} \times 5 \mathrm{~mm} \times 1 \mathrm{~mm}$ ) was surrounded by the copper coil. The temperature evolution was monitored using proportional-integral-derivative (PID) controller. During the experiment, the total time was calculated from room temperature to $670{ }^{\circ} \mathrm{C}$ and $670{ }^{\circ} \mathrm{C}$ to room temperature. The estimated average heating and cooling rates were calculated from the heating and cooling temperatures respectively. It has to be noted that the heating and cooling rates are the average heating and cooling rates as the heating and cooling rate was not uniform throughout the temperature range. The approximate heating and cooling rates were $6{ }^{\circ} \mathrm{C} / \mathrm{s}$ and $2{ }^{\circ} \mathrm{C} / \mathrm{s}$, respectively. The detailed in-situ heating-cooling schedule is given in Fig. 1a. The diffraction patterns were recorded during heating, holding and cooling of the sample. The schematic representation of the diffraction geometry is plotted in Fig. 1b. The x-ray diffraction patterns were taken at an interval temperature of $\sim 50{ }^{\circ} \mathrm{C}$ and the acquisition time was less than a second.

The microstructural features, incorporating the orientation, were characterized using the electron back-scatter diffraction (EBSD) technique; it was performed in FEI-Sirion FEG-SEM system, operating at $30 \mathrm{kV}$ with a step size of $100 \mathrm{~nm}$. The EBSD data acquisition and analysis were carried out using the TSL-OIM ${ }^{\mathrm{TM}}$ software, considering a minimum boundary misorientation of $2^{\circ}$. The EBSD scan was carried out at the transverse (TD) plane. The elemental mapping, using electron

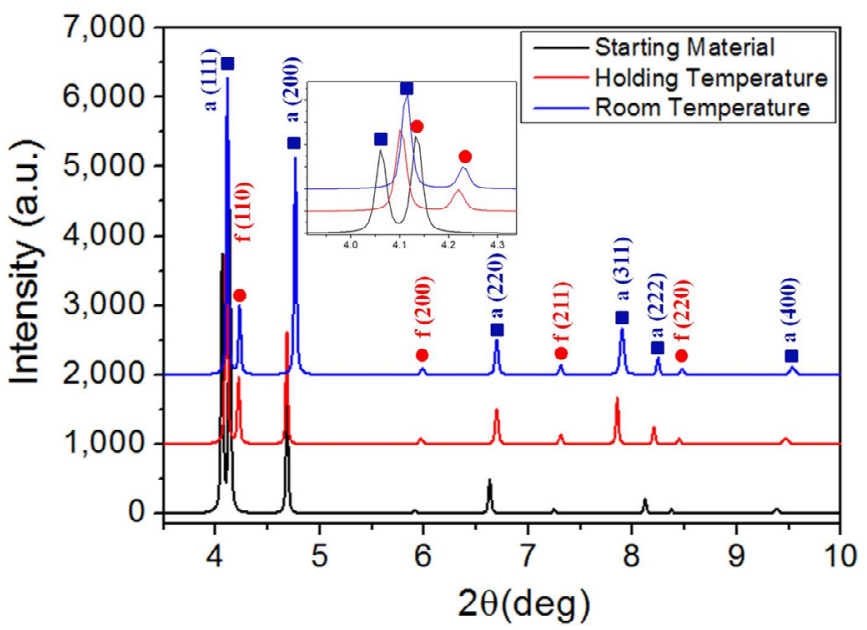

Fig. 2. Diffraction patterns showing peak position and intensity for starting material, holding temperature and room temperature. The inset shows the enlarged portion of $2 \theta, 3-5^{\circ}$.

probe micro-analysis (EPMA) was used for compositional analysis and to study the elemental distribution in the inter-critical annealed sample. A JEOL JXA-8530F instrument was used for the composition analysis.

\section{Results}

Fig. 2 shows the diffraction patterns that were obtained from the starting material, at the inter-critical temperature and the room temperature (after cooling). For brevity, only three patterns (starting sample, during holding and after cooling to room temperature) are shown below. The patterns show the presence of austenite and ferrite phases. A change in intensity of the peaks corresponding to both the phases was observed during heating and cooling, which indicates changes in the phase fraction of the alloy during the experiment.

In addition to changes in intensity, the peaks got shifted as a result of annealing treatment (inset of Fig. 2). Fig. 3a depicts the relative changes in d-spacing during heating to ICT, holding and then cooling. It can be seen that there is very small change in the d-spacing for both the austenite and ferrite phases during the initial stage of annealing. Subsequently, there is a gradual increase in the d-spacing for the austenite phase and a rather steep decrease in the d-spacing of the ferrite phase. The d-spacing for both the phases do not change on cooling. The 
(a)

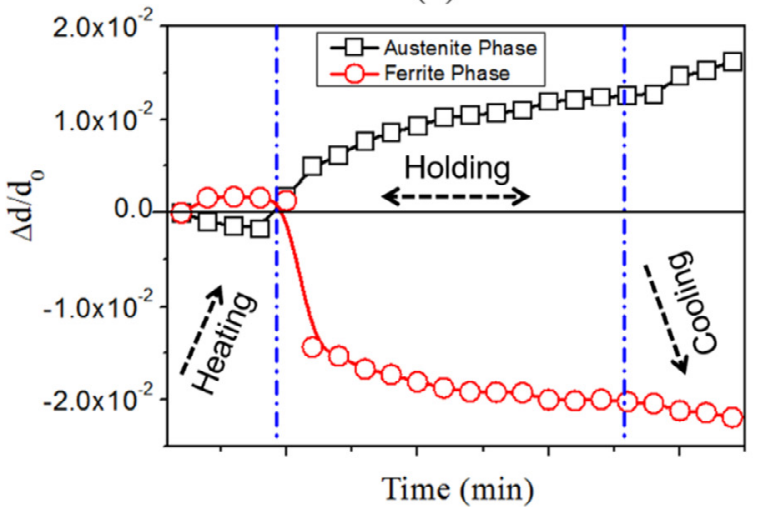

(b)

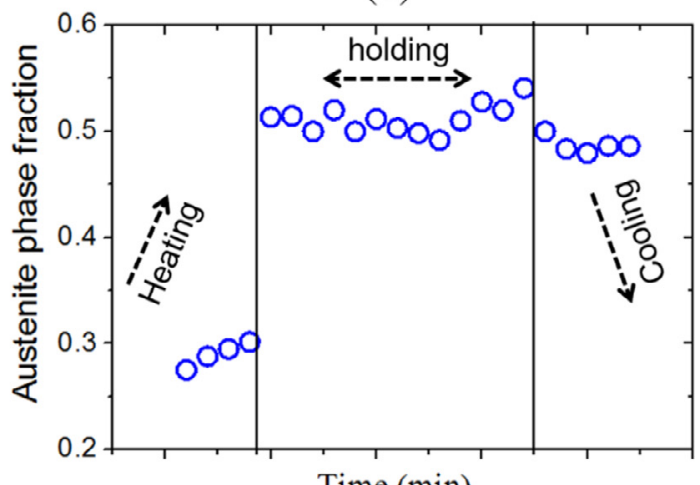

Time (min)

Fig. 3. (a) Relative changes in d-spacing $\left(\Delta d / d_{0}\right.$ and (b) evolution of austenite phase fraction during heating, holding at ICT temperature and cooling.

(a)
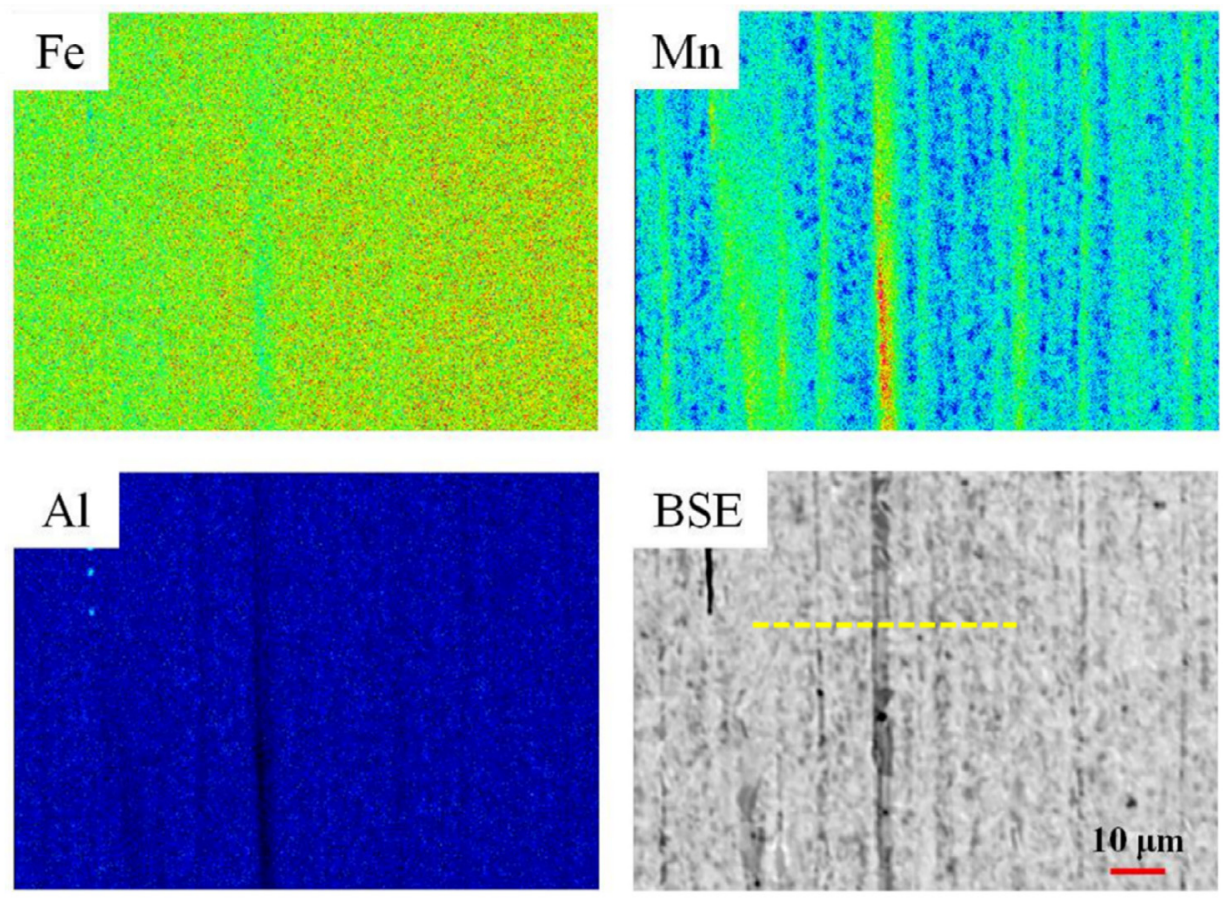

(b)
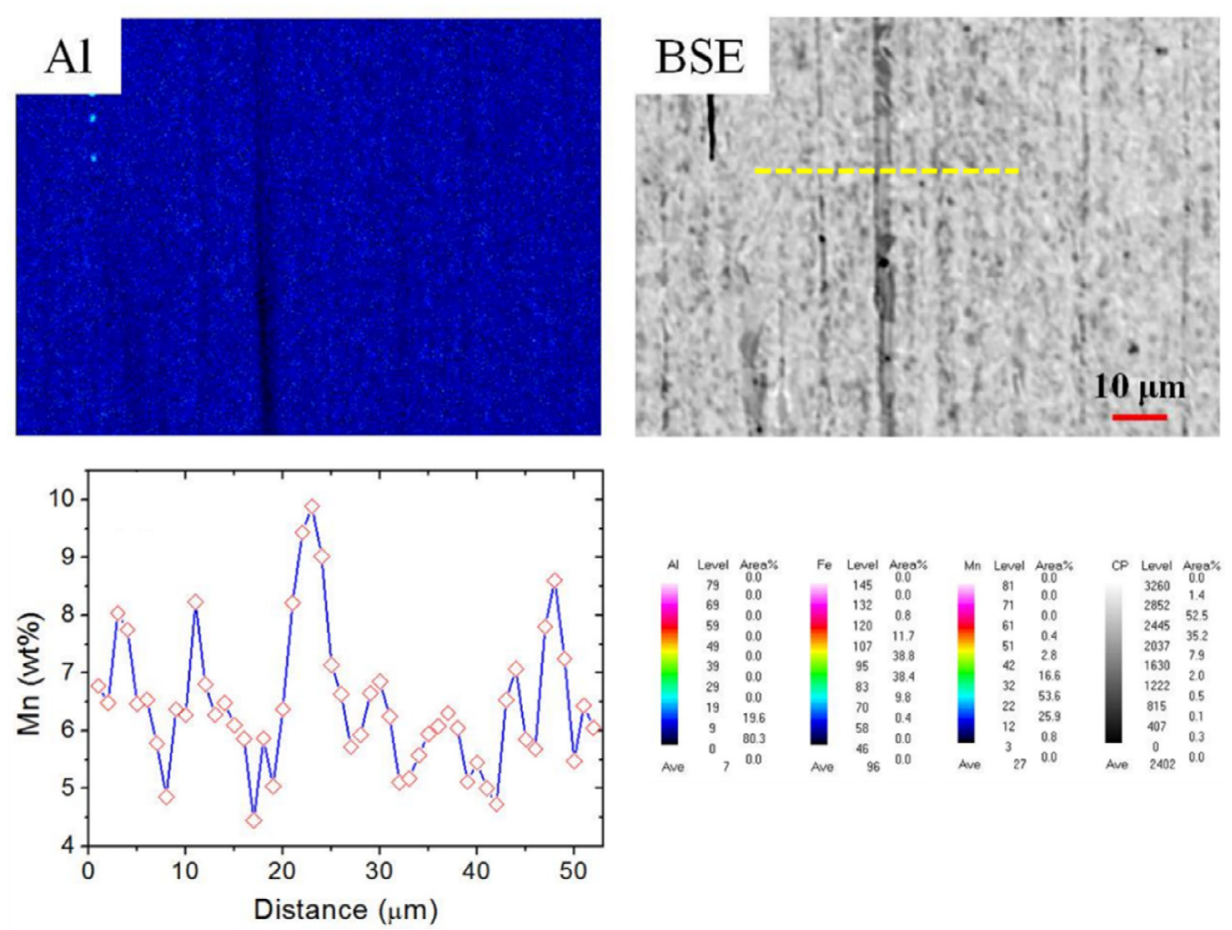

Fig. 4. (a) Elemental distribution map (for $\mathrm{Fe}, \mathrm{Mn}$ and $\mathrm{Al}$ ) combined with back-scatter electron (BSE) image. (b) Mn concentration across the line, marked with yellow color in Fig. (a) BSE image. determination of phase fraction reveals that the austenite fraction increases to $50 \%$ during inter-critical annealing and the austenite content of the microstructure remains stable at this level even after cooling (Fig.3b).

The elemental distribution map in Fig. 4a shows a large partitioning of Mn after inter-critical annealing. The microstructure shows the formation of branded Mn-rich and Mn-lean regions. The Mn rich regions are austenite phase, where enough $\mathrm{Mn}$ is available for stabilization of the austenite phase. However, in Mn-lean regions, the presence of Mn is not enough for stabilization of the austenite phase; so, the Mn-lean regions will be ferrite phase. The presence of Mn-rich and Mn-lean regions makes a banded or lamellar phase morphology, where the austenite and ferrite phases are aligned in a lamellar fashion. Fig. 4b shows the Mn concentration across the red line (marked in the BSE image in Fig. 4a). 

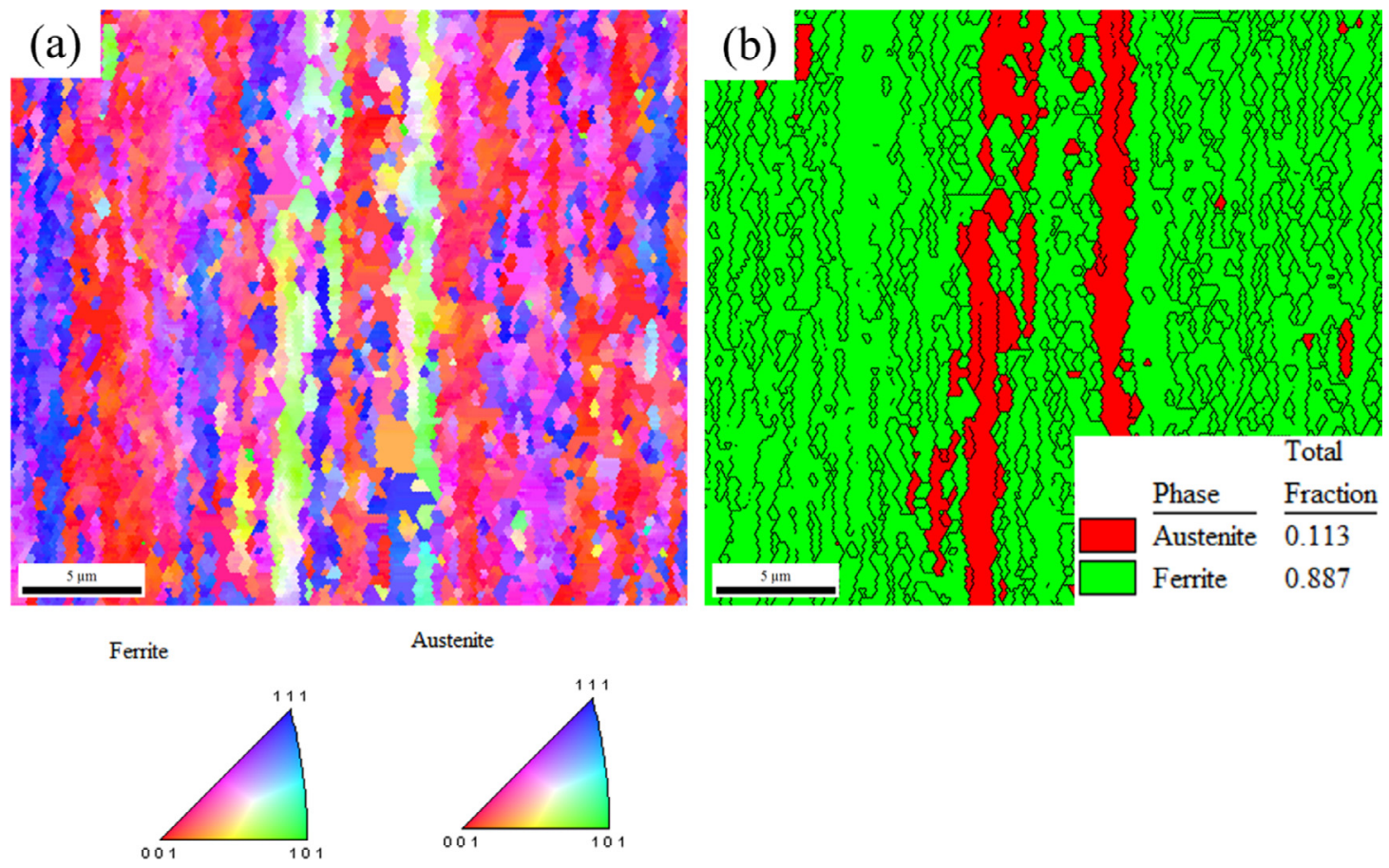

Fig. 5. EBSD generated (a) IPF map and (b) phase map of the starting annealed sample.

The concentration profile shows the inhomogeneous distribution of Mn (4.5 to $10 \mathrm{wt} . \%$ ) across the line. Lee et al. [21] showed a similar Mn-rich austenite region in Fe-0.1C-10Mn-1Si-0.3Mo-0.5 V steel. The presence of sufficient amounts of Mn and C in the banded regions at the inter-critical annealing temperature favours a high fraction of austenite phase stability at room temperature. It important to note here that after casting, the specimen was hot rolled prior to inter-critical annealing in order to eliminate the possible segregation of Mn (during casting). Further, it is more likely that during hot rolling Mn was dissolved more or less homogeneously throughout the specimen, as the solubility of $\mathrm{Mn}$ in $\mathrm{Fe}$ is higher in the austenite.

Fig. 5a and 5b show the hot-rolled microstructure prior to the in-situ experiment. The microstructure shows the presence of $\sim 11 \%$ austenite phase (calculated from the 2D EBSD phase map) and the phase distribution is lamellar. Fig. 6a displays the microstructure of the annealed sample after ICT treatment. The microstructure consists of $\sim 60 \%$ austenite phase (Fig. $6 \mathrm{~b}$ ). The austenite phase is characterized by a lamellar morphology; such an aligned morphology is a result of very fast segregation of $\mathrm{Mn}$ and $\mathrm{C}$ atoms during short-time annealing. The hot-rolled microstructure shows the presence of lamellar phase morphology which can also be an effect in lamellar phase alignment after inter-critical annealing. The presence of equiaxed microstructure indicates that both the phases are recrystallized during annealing at ICT. The resulting microstructure after dynamic restoration is typified by a fine grain structure consisting of austenite and ferrite (Fig. 6a). The average grain size was measured as $1.8 \mu \mathrm{m}$ and $0.8 \mu \mathrm{m}$ for the austenite and ferrite phases, respectively, after inter-critical annealing.

In the final annealed microstructure, large fractions of annealing twins (marked with arrow) were observed in the austenite grains (Fig. 6a). The misorientation distribution plots, corresponding to the austenite and ferrite phases are displayed in Fig. 6c. A rather small fraction of low angle grain boundaries in the austenite phase suggests the completion of recrystallization of the austenitic phase during short time annealing.

A very high frequency of misorientation was observed at $\sim 43^{\circ}$ for both the phases. The peak at $\sim 43^{\circ}$ in the misorientation distribution corresponds to the common misorientation angle between austenite and ferrite phases. The sharp maximum in the misorientation distribution at $\sim 60^{\circ}$ in the austenite phase is attributed to the presence of annealing twins in the microstructure [25]. The texture is presented by the (111) and (110) pole figures for the austenite and ferrite phases respectively (Fig. 6d). The locations of intensity maxima in the (111) pole figure for the austenite phase and those in the (110) pole figure for the ferrite phase coincide, which is an indication of adherence to the orientation relationship during annealing: $\left.\left.\{111\}_{\gamma} \|\{110\}_{\alpha} ;<110\right\rangle_{\gamma} \|<111\right\rangle_{\alpha}$. This relationship indicates that the close packed plane of the austenite phase is parallel to the close packed plane of the ferrite phase.

\section{Discussion}

During the initial stage of heating, the change in d-spacing could be due to relaxation of the residual stresses that had accumulated during rolling (Fig. 3a). During holding at a high temperature, the relative change in d-spacing can be explained by the change in the lattice parameter due to change in composition during annealing. At the inter-critical temperature, the $\mathrm{Mn}$ and $\mathrm{C}$ atoms move from the ferrite phase to the austenite phase; as a result the concentrations of these elements in the austenite phase increases. The enrichment by more Mn and $\mathrm{C}$ atoms increases the lattice parameter in the austenite phase and depletion reduces lattice parameter in ferrite phase (Fig. 3a). The increased concentrations of $\mathrm{Mn}$ and $\mathrm{C}$ atoms significantly increases phase fraction of austenite. The partitioned $\mathrm{Mn}$ and $\mathrm{C}$ atoms in the austenite phase also increases the stacking SFE towards the higher side. Due to increase in the SFE deformation is possible by twinning instead of strain-induced martensite transformation [16]. The Rapid segregation of Mn creates Mn-rich and Mn-lean regions. The relative decrease in d-spacing $\left(\Delta \mathrm{d} / \mathrm{d}_{0}\right)$ for the ferrite phase indicates that Mn and C flow towards the austenitic phase. Simultaneously the increase in lattice parameter also indicates enrichment of $\mathrm{Mn}$ and $\mathrm{C}$ during annealing. Elemental partitioning during short-time annealing at ICT temperature is reported in References [17,18]. De Cooman [2] also reported Mn partitioning in the Fe-7Mn-0.09C-1.3Si alloy after annealing at $600{ }^{\circ} \mathrm{C}$ and $650{ }^{\circ} \mathrm{C}$ for $168 \mathrm{~h}$, which led to a stabilization of $39 \%$ and $47 \%$ austenite, respectively. Therefore, it is well-established that Mn and C play major role in stabilizing the retained austenite during inter-critical annealing. However, the stability of the austenite phase is attributed to 

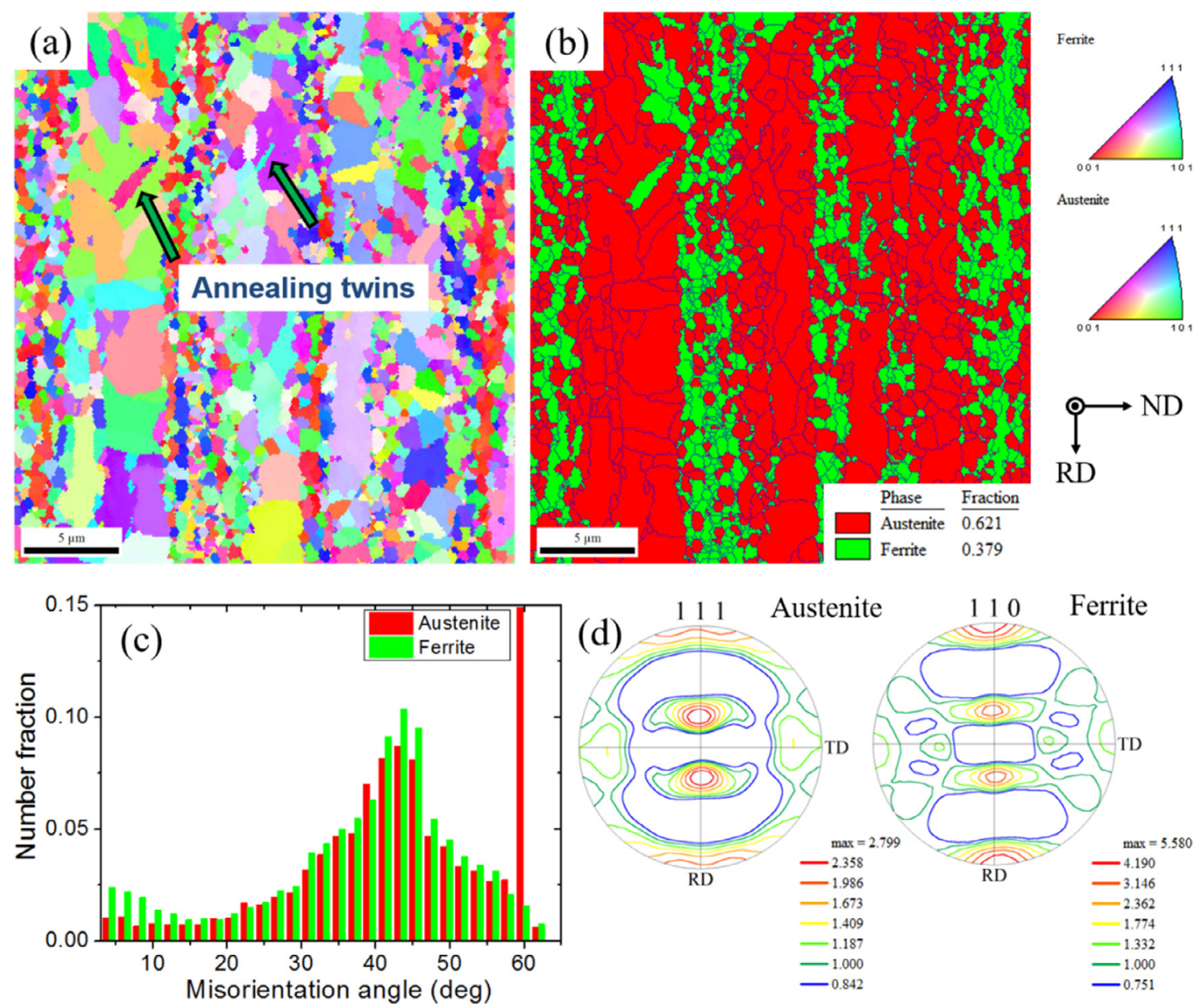

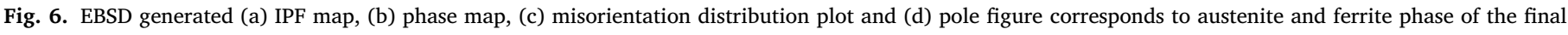
annealed sample.

the equilibrium solubility of $\mathrm{Mn}$ in the microstructure during annealing. The Mn and C get partitioned during short-time annealing and lead to a higher fraction of retained austenite in the microstructure. The $\mathrm{Mn}$ atoms diffuse rapidly into growing austenite grains due to the large difference in solubility of $\mathrm{Mn}$ in the austenite and ferrite phases. Further, the $\mathrm{C}$ atoms diffuse relatively faster compared to $\mathrm{Mn}$ due to smaller atomic size. Shen et al. [4] reported that the partitioning of $\mathrm{C}$ from the martensite to the austenite phase in Fe-1.7Mn-0.2C alloy was 1.1-1.85 wt.\% during annealing for very short time. The solubility of $\mathrm{C}$ is higher in austenite than in ferrite. Therefore, it is expected that the $\mathrm{C}$ concentration will be higher in the austenite phase. The segregation of $\mathrm{Mn}$ and $\mathrm{C}$ is responsible for the stabilization of austenite at room temperature. Lee et al. $[18,19]$ reported $\mathrm{Mn}$ and C partitioning during annealing at $680{ }^{\circ} \mathrm{C}$ for $3 \mathrm{~min}$. The authors emphasize that $8 \mathrm{wt} . \%$ of $\mathrm{Mn}$ and a significant amount of $\mathrm{C}$ get partitioned during annealing for such a short time to stabilize the austenitic phase at room temperature.

Hence, the concept of mechanical stability can be completely avoided where the presence of a very high dislocation density stops the growth of martensite embryos and stabilizes the austenitic phase [24].

In the present investigation, phase fractions of the austenite and ferrite phases in the starting material and the corresponding changes during heating and cooling were calculated using the direct comparison method [20]. The Fig. 3b shows the austenite phase fraction evolution during heating, holding and cooling. In the direct comparison method, the (111) and (200) peaks for austenite phase, and the (110) and (200) peaks for ferrite phase were used for phase fraction calculation. The starting material was characterized by $\sim 25-30 \%$ of the austenite phase. After annealing, the austenite phase fraction increased to $50-55 \%$. The most important observation is that during cooling, the austenite phase fraction remained unchanged up to room temperature. This suggests that there was no reverse flow of $\mathrm{Mn}$ and $\mathrm{C}$ atoms, or any reverse phase transformation during cooling.

During inter-critical annealing, grain growth was restricted as grain growth is dependent on inter-phase interface mobility (interface between the austenite and ferrite phases) and the recrystallization temperature. In general, it is likely that the energy associated with interface mobility is higher than the grain boundary mobility; as a result, grain growth was not observed in both the phases. The inhomogeneous distribution of the austenite phase is due to the anisotropic mobility of $\mathrm{Mn}$ and $\mathrm{C}$ atoms during short time annealing.

The distribution of $\mathrm{Mn}$ in an inter-critical annealed sample is shown in Fig. 4a. The elemental distribution map indicates an inhomogeneous distribution of Mn atoms throughout the microstructure, where Mn-rich and Mn-lean regions were observed. This could be due to inhomogeneous or anisotropic partitioning of $\mathrm{Mn}$, where partitioning depends on the anisotropic mobility of $\mathrm{Mn}$ and $\mathrm{C}$ atoms during annealing. In addition to the elemental partitioning of $\mathrm{Mn}$ and $\mathrm{C}$, factors such as grain size and plastic strain also affect the stability of the metastable austenite phase $[18,19,22]$. In the present case, strain accumulation during rolling has a strong influence on the partitioning of $\mathrm{Mn}$ and $\mathrm{C}$ atoms. The atomic diffusivity is faster in the regions of high accumulated plastic strain compared to low plastic strain regions, which further introduces inhomogeneous austenitic phase distribution in the microstructure. Yang and Bhadeshia [23] have reported that the prior austenitic grain size also contributes to the austenitic phase stability at room temperature. In the present case, the plastic strain in the 
microstructure was high before inter-critical annealing and the prior austenitic grain size was in the sub-micrometer range which could also play a crucial role in higher $\mathrm{Mn}$ and $\mathrm{C}$ partitioning.

The experimental results suggest that the austenite phase fraction, as predicted by Thermo-Calc is very close to the experimentally observed austenite phase fraction that the retained phase fraction of austenite at room temperature can be tuned by changing the inter-critical annealing temperature. Since the phase fraction of retained austenite plays an important role in TRIP steels for maintaining a good balance between strength and ductility [26], the findings of the present investigation are of immense significance in tailoring the properties of medium Mn TWIP/TRIP steels.

\section{Summary and conclusions}

In the present investigation, the evolution of the austenite phase during heating, holding at the inter-critical temperature and subsequent cooling had been investigated by in-situ high energy synchrotron diffraction experiment. During the in-situ experiment, relative changes in d-spacing during heating, holding and cooling are attributed to the elemental partitioning and hence the stability of austenite phase during inter-critical annealing. The resulting microstructure clearly reveals the presence of $\sim 50 \%$ austenite phase at the inter-critical temperature and it remains stable even after cooling down to room temperature. Experimentally observed austenite phase fraction is very close to phase fraction predicted by Thermo-Calc. The partitioned elements, Mn and $\mathrm{C}$, during inter-critical annealing are responsible for the change in the lattice parameter as well as increase in the austenite phase fraction. The final microstructure is an austenite-ferrite microstructure with a fine grained structure of austenite and ferrite phases, where the average grain sizes are $1.8 \mu \mathrm{m}$ and $0.8 \mu \mathrm{m}$ respectively. The fine grain microstructure is a result of very short term annealing and lower intercritical annealing temperature. The morphology of the microstructural constituents is lamellar with alternate layers of austenite and ferrite phases, where both the phases follow crystallographic orientation relationship. The combined effect of strain accumulation during rolling as well as the elemental partitioning during inter-critical annealing plays crucial role for development of such a microstructure.

\section{Decleration of Competing Interest}

The authors declare that they have no known competing financial interests or personal relationships that could have appeared to influence the work reported in this paper.

\section{Acknowledgements}

This work was funded by a grant from R\&D Division, Tata Steel India Ltd (Grant number: PC-99-236). The Deutches Elektronen-Synchrotron (DESY), Hamburg synchrotron radiation facility is acknowledged for providing P07 Beamline, Petra III under the proposal I-20160573. The extensive use of microscopes at the Advanced Facility of Microscopy and Microanalysis (AFMM) facility, Indian Institute of Science, Bangalore is also acknowledged.

\section{Supplementary material}

Supplementary material associated with this article can be found, in the online version, at doi:10.1016/j.mtla.2020.100594.

\section{References}

[1] D.K. Matlock, J.G. Speer, Third generation of AHSS: microstructure design concepts, in: Microstructure and Texture in Steels, Springer, 2009, pp. 185-205.

[2] B.C.De Cooman, P. Gibbs, S. Lee, D.K. Matlock, Transmission electron microscopy analysis of yielding in ultrafine-grained medium Mn transformation-induced plasticity steel, Metall. Mater. Trans. A 44 (2013) 2563-2572.

[3] J.G. Speer, D.V. Edmonds, F.C. Rizzo, D.K. Matlock, Partitioning of carbon from supersaturated plates of ferrite, with application to steel processing and fundamentals of the bainite transformation, Curr. Opin. Solid State Mater. Sci. 8 (2004) 219-237.

[4] Y.F. Shen, Y.D. Liu, X. Sun, Y.D. Wang, L. Zuo, R.D.K. Misra, Improved ductility of a transformation-induced-plasticity steel by nanoscale austenite lamellae, Mater. Sci. Eng. A 583 (2013) 1-10.

[5] E.J. Seo, L. Cho, B.C. DeCooman, Application of quenching and partitioning processing to medium Mn steel, Metall. Mater. Trans. A 46 (2015) 27-31.

[6] S. Lee, B.C. DeCooman, On the selection of the optimal intercritical annealing temperature for medium Mn trip steel, Metall. Mater. Trans. A 44 (2013) 5018-5024.

[7] J. Han, Y.-K. Lee, The effects of the heating rate on the reverse transformation mechanism and the phase stability of reverted austenite in medium Mn steels, Acta Mater. 67 (2014) 354-361

[8] A. Arlazarov, M. Gouné, O. Bouaziz, A. Hazotte, G. Petitgand, P. Barges, Evolution of microstructure and mechanical properties of medium Mn steels during double annealing, Mater. Sci. Eng. A 542 (2012) 31-39.

[9] D. Raabe, S. Sandlöbes, J. Millán, D. Ponge, H. Assadi, M. Herbig, P.-P. Choi, Segregation engineering enables nanoscale martensite to austenite phase transformation at grain boundaries: a pathway to ductile martensite, Acta Mater. 61 (2013) 6132-6152.

[10] Yu Li, Wei Li, Wenqing Liu, Xiaodong Wang, Xueming Hua, Huibin Liu, Xuejun Jin, The austenite reversion and co-precipitation behavior of an ultra-low carbon medium manganese quenching-partitioning-tempering steel, Acta Mater. 146 (2018) 126-141.

[11] Z.C. Li, H. Ding, R.D.K. Misra, Z.H. Cai, Microstructure-mechanical property relationship and austenite stability in medium-Mn trip steels: the effect of austenite-reverted transformation and quenching-tempering treatments, Mater. Sci. Eng. A 682 (2017) 211-219.

[12] D.-W. Suh, S.-J. Park, T.-H. Lee, C.-S. Oh, S.-J. Kim, Influence of Al on the microstructural evolution and mechanical behavior of low-carbon, manganese transformation-induced-plasticity steel, Metall. Mater. Trans. A 41 (2010) 397-408.

[13] S. Lee, K. Lee, B.C. DeCooman, Observation of the Twip + Trip plasticity-enhancement mechanism in Al-added 6áWtáPct medium Mn steel, Metall. Mater. Trans. A 46 (2015) 2356-2363.

[14] Thermo-Calc, Software Users Guide, Version S, Stockholm Technology Park, Stockholm, (2008)

[15] A. Hammersley, FIT2D software: http://www.esrf.eu/computing/scientific.

[16] O. Bouaziz, S. Allain, C.P. Scott, P. Cugy, D. Barbier, High manganese austenitic twinning induced plasticity steels: a review of the microstructure properties relationships, Curr. Opin. Solid State Mater. Sci. 15 (2011) 141-168.

[17] F. HajyAkbary, J. Sietsma, R.H. Petrov, G. Miyamoto, T. Furuhara, M.J. Santofimia, A quantitative investigation of the effect of Mn segregation on microstructural properties of quenching and partitioning steels, Scripta Mater. 137 (2017) 27-30.

[18] S. Lee, S.-J. Lee, B.C. De Cooman, Austenite stability of ultrafine-grained transformation-induced plasticity steel with Mn partitioning, Scripta Mater. 65 (2011) 225-228.

[19] S.-J. Lee, S. Lee, B.C. De Cooman, Mn partitioning during the intercritical annealing of ultrafine-grained $6 \% \mathrm{Mn}$ transformation-induced plasticity steel, Scripta Mater. 64 (2011) 649-652.

[20] B.D. Cullity, J.W. Weymouth, Elements of X-ray diffraction, Am. J. Phys. 25 (1957) 394-395.

[21] H. Lee, M.C. Jo, S.S. Sohn, A. Zargaran, J.H. Ryu, N.J. Kim, S. Lee, Novel medium-Mn (austenite+ martensite) duplex hot-rolled steel achieving 1.6GPa strength with $20 \%$ ductility by Mn-segregation-induced trip mechanism, Acta Mater. 147 (2018) 247-260.

[22] K. Tomimura, S. Takaki, Y. Tokunaga, Reversion mechanism from deformation induced martensite to austenite in metastable austenitic stainless steels, ISIJ Int. 31 (1991) 1431-1437.

[23] H.-S. Yang, H. Bhadeshia, Austenite grain size and the martensite-start temperature, Scripta Mater. 60 (2009) 493-495.

[24] J. Breedis, Influence of dislocation substructure on the martensitic transformation in stainless steel, Acta Metall. 13 (1965) 239-250.

[25] H. Beladi, I.B. Timokhina, Y. Estrin, J. Kim, B.C. De Cooman, S.K. Kim, Orientation dependence of twinning and strain hardening behaviour of a high manganese twinning induced plasticity steel with polycrystalline structure, Acta Mater. 59 (2011) 7787-7799.

[26] Z.J. Xie, G. Han, W.H. Zhou, X.L. Wang, C.J. Shang, R.D.K. Misra, A novel multi-step intercritical heat treatment induces multi-phase microstructure with ultra-low yield ratio and high ductility in advanced high-strength steel, Scripta Mater. 155 (2018) $164-168$. 